\title{
Dehydroepiandrosterone (DHEA)
} Therapy is a promising policy for Infertile Aged Women to get Spontaneous Conception

\author{
Abd-Elhaseib Salah* \\ ${ }^{1}$ Department of Obstetrics and Gynecology, Menoufia University, Egypt
}

Submission: April 12, 2019 , Published: August 31, 2019

*Corresponding author: Abd-Elhaseib Salah, Department of Obstetrics and Gynecology Faculty of Medicine, Menoufia University, Shibin El-Kom City, Menoufia governorate, Egypt

\begin{abstract}
Objectives: Evaluating the clinical pregnancy rate (CPR) after priming of infertile women older than 38 years and have poor ovarian reserve using dehydroepiandrosterone (DHEA).

Patients \& Methods: The study included 282 fulfilling inclusion criteria received DHEA (50 mg twice daily for 3 months) and were allowed to get spontaneous pregnancy. Women who failed to get pregnant received oral clomiphene citrate $100 \mathrm{mg}$ for five days starting on day 3 of the menstrual cycle) followed by daily intramuscular injection of $150 \mathrm{IU}$ of human menopausal gonadotropin till ovulation was assured by TVU. Evaluated parameters included bilateral ovarian antral follicular count and hormonal profile were estimated at time of enrollment and at end of each 3-m phases. Chemical and clinical pregnancy rates and abortion rate.
\end{abstract}

Results: Clinical pregnancy and continued pregnancy till 2nd trimester rates were $6.7 \%$ and $5.7 \%$ for Phase- 1 and for Phase- 2 were $16.3 \%$ and $14.4 \%$, respectively. Collectively, chemical pregnancy rate was $26.2 \%$, CPR was $21.6 \%$, abortion rate was $13.1 \%$ and rate of continued pregnancy till 2 nd trimester was $18.8 \%$. CPR with induction after 3-m DHEA priming was significantly $(\mathrm{p}=0.00039)$ higher than without induction. The applied protocol allowed getting pregnancy that was continued till 2nd trimester in $71.6 \%$ of women who had positive chemical pregnancy and $86.9 \%$ of women who had clinical pregnancy. Moreover, DHEA therapy induced significant reduction of serum FSH, LH with significant increase of testosterone, E2 and AMH levels with subsequently higher AFC compared to pretreatment levels.

Conclusion: Priming of infertile aged women with POR using DHEA with or without ovulation induction improves the chance of spontaneous conception and spares the need for assisted reproduction procedures by $21.6 \%$ of treated women. Ovulation induction after DHEA priming triples the chance for spontaneous conception.

Keywords: Infertile aged women; DHEA priming; Spontaneous conception; Ovulation induction

Abbreviations: POF: Premature Ovarian Failure; AMH: Anti-Müllerian Hormone; FSH: Follicle-Stimulating Hormone; AFC: Antral Follicle Count; DHEA: Dehydroepiandrosterone; CPR: Clinical Pregnancy Rate; TUV: Transvaginal Ultrasonography; AMH: Anti-Müllerian Hormone; POR: Poor Ovarian Reserve; CPR: Chemical and Clinical Pregnancy Rates

\section{Introduction}

Ovarian ageing shows great inter-individual variability, is characterized by quantitative and qualitative alteration of ovarian oocyte reserve and about $20 \%$ of infertile women show signs of premature ovarian ageing [1]. Premature ovarian failure (POF) may be considered as autoimmune disease secondary to absence of ovarian immunological protection and detection of ovarian autoantibodies strongly support this hypothesis [2]. POF may have a genetic etiopathogenesis as the presence of breast cancer susceptibility gene (BRCA) mutation was found to be linked to

accelerated ovarian aging and reduced ovarian reserve [3]. Inflammatory aging refers to a chronic and low-degree proinflammatory status with increasing age and is closely associated with multiple diseases, as POF [4].

Anti-Müllerian hormone (AMH) is a homo-dimeric glycoprotein produced by granulosa cells of growing ovarian follicles and has an inhibitory effect on primordial follicle recruitment and response of growing follicles to follicle-stimulating hormone (FSH) [5]. AMH levels may predict the ovarian response to ovarian hy- 
perstimulation for IVF, timing of menopause, iatrogenic damage to the ovarian follicle reserve and as surrogate for antral follicle count (AFC) [6]. Also, for prediction of the ovarian response to gonadotropin therapy, serum AMH levels are stronger predictor than ultrasonographic AFC [7].

Dehydroepiandrosterone (DHEA) is a 19-carbon steroid and its actions are partially exerted through its metabolites [8]. DHEA acts as prohormone, endogenous precursor of ovarian and adrenal androgens and a metabolic intermediate in ovarian follicular steroidogenesis [9]. Metabolically, DHEA affects oocyte metabolism and its elevated systemic levels may contribute to poor pregnancy outcomes in women with hyperandrogenism [10].

\section{Hypothesis}

Considering the fact that androgens can influence ovarian follicular growth, augment steroidogenesis, promote follicular recruitment and increase number of primary and pre-antral follicles [11]. The current study hypothesized that priming of infertile women with poor ovarian reserve using dehydroepiandrosterone (DHEA) may improve clinical pregnancy rate (CPR) in women older than 38 years seeking for pregnancy.

\section{Setting}

Obstetrics \& Gynecology Department, Menofea University Hospital

\section{Design}

Prospective two-stage clinical trial

\section{Patients \& Methods}

The current study was conducted since March 2016 till Oct 2018 to allow a minimum follow-up period of 6 months for the last case enrolled in the study. All women in age range of 38-40 years and complaining of failure to get spontaneous pregnancy since more than one-year despite of the regular intercourse, no use of contraceptives, and absence of male factor for infertility. Women with endocrinopathy, tubal factor, had previous attempts of assisted reproduction, presence of male factor, uterine abnormalities, had diabetes mellitus, renal or cardiac disease were excluded from the study to equalize enrolment criteria.

All women eligible for evaluation undertook full clinical examination, transvaginal ultrasonography (TUV), hystrosalpingography, and gave blood samples for evaluation of hormonal profile. Husbands were asked to perform seminal analysis for assurance of fertility and were sent for andrology clinic for eval-

Table 1: Patients' enrolment data.

\begin{tabular}{|c|c|c|}
\hline \multicolumn{2}{|c|}{ Data } & Findings \\
\hline \multirow{3}{*}{ BMI data } & Age (years) & $40.1 \pm 3.6$ \\
\cline { 2 - 3 } & Body weight $(\mathrm{kg})$ & $80.9 \pm 6.6$ \\
\cline { 2 - 3 } & Body height $(\mathrm{cm})$ & $169 \pm 3.7$ \\
\hline \multirow{2}{*}{ BMI $\left(\mathrm{kg} / \mathrm{m}^{2}\right)$} & $28.4 \pm 2.6$ \\
\hline
\end{tabular}

uation of potency sufficient to commit intercourse. Enrolment criteria included primary or secondary infertility for $>1$-year duration, serum anti-Müllerian hormone (AMH) level of $\leq 1 \mathrm{ng}$ / $\mathrm{ml}$, and ultrasonographic manifestations of poor ovarian reserve (POR). Enrolled couples signed written fully informed consent prior to enrolment. Diagnostic criteria for poor ovarian reserve included old age or presence of other risk factor for POR, history of previous POR, abnormal ovarian reserve including low AFC and/or AMH [12].

\section{Study protocol}

The study protocol comprised two-phase, during Phase-1, all enrolled women received DHEA therapy in a dose of $50 \mathrm{mg}$ twice daily for three months and women were allowed to get the trial for getting spontaneous conception. Women who failed to get pregnant underwent induction of ovulation using $100 \mathrm{mg}$ of oral clomiphene citrate (Clomifene; Young Poong Pharmaceutical, Incheon, Korea) for five days starting on day 3 of the menstrual cycle, followed by daily intramuscular injection of $150 \mathrm{IU}$ of human menopausal gonadotropin (Pregnyl; Organon, Oss, the Netherlands) till ovulation was assured by TVU.

I. Bilateral ovarian AFC using TVU on day 2-3 of cycle by counting number of antral follicles reaching $2-10 \mathrm{~mm}$ in transverse axial section of each ovary and count was summed. AFC was determined at time of enrollment, after DHEA therapy (End of Phase-1), and after induction of ovulation (End of Phase-2).

II. Hormonal profile was re-checked at the end Phase-1 and -2 .

III. Chemical and clinical pregnancy rates (CPR) and abor-

\section{Statistical analysis}

Obtained data were presented as mean \pm SD, numbers and percentages. Results were analyzed using paired t-test and Oneway ANOVA Test. and Chi-square test (X2 test). Statistical analysis was conducted using the IBM SPSS (Version 23, 2015) for Windows statistical package. P value $<0.05$ was considered statistically significant.

\section{Results}

The study included 341 infertile women eligible for evaluation, 59 women were excluded for not fulfilling inclusion criteria and 282 women were included in the study (Figure 1); inclusion criteria were shown in Table 1, Table 2.

\section{Evaluated parameters} tion rate 


\section{Journal of Gynecology and Women's Health}

\begin{tabular}{|c|c|c|c|c|}
\hline \multirow{3}{*}{ Infertility } & \multirow{3}{*}{ Type } & \multirow{3}{*}{ Secondary } & Primary & $92(32.6 \%)$ \\
\cline { 3 - 4 } & & & 1-offspring & $121(42.9 \%)$ \\
\cline { 3 - 4 } & & 2-offspring & $69(24.5 \%)$ \\
\cline { 3 - 4 } & & Mean number & $1.4 \pm 0.5$ \\
\cline { 3 - 4 } & & \multicolumn{2}{|c|}{ Duration (years) } & $3.7 \pm 2.3$ \\
\hline
\end{tabular}

Data are presented as mean $\pm \mathrm{SD}$, numbers \& percentages; BMI: Body mass index

Table 2: Estimated hormonal levels and TUV detected AFC of studied patients at end of phase-1 compared to measures at time of enrolment.

\begin{tabular}{|c|c|c|c|}
\hline Hormones & At time of enrolment & 3-m after enrolment & P value \\
\hline FSH (mIU/ml) & $16.45 \pm 2.8$ & $15.8 \pm 3.2$ & 0.021 \\
\hline LH (mIU/ml) & $7.6 \pm 3.25$ & $6.82 \pm 4.38$ & 0.012 \\
\hline E2 (pg/ml) & $87.86 \pm 35.6$ & $96 \pm 55.9$ & 0.039 \\
\hline Testosterone (ng/ml) & $0.77 \pm 0.49$ & $0.82 \pm 0.36$ & 0.011 \\
\hline AMH (ng/ml) & $0.98 \pm 0.41$ & $1.18 \pm 0.79$ & 0.001 \\
\hline AFC (follicle) & $4.16 \pm 1.54$ & $4.62 \pm 2.95$ & 0.021 \\
\hline
\end{tabular}

Data are presented as mean \pm SD; FSH: Follicle stimulating factor; LH: Luteinizing hormone; E2: Estradiol; AMH: anti-Müllerian hormone; AFC: Antral follicle count; $P$ value indicates difference between both groups; $p<0.05$ indicates significant difference

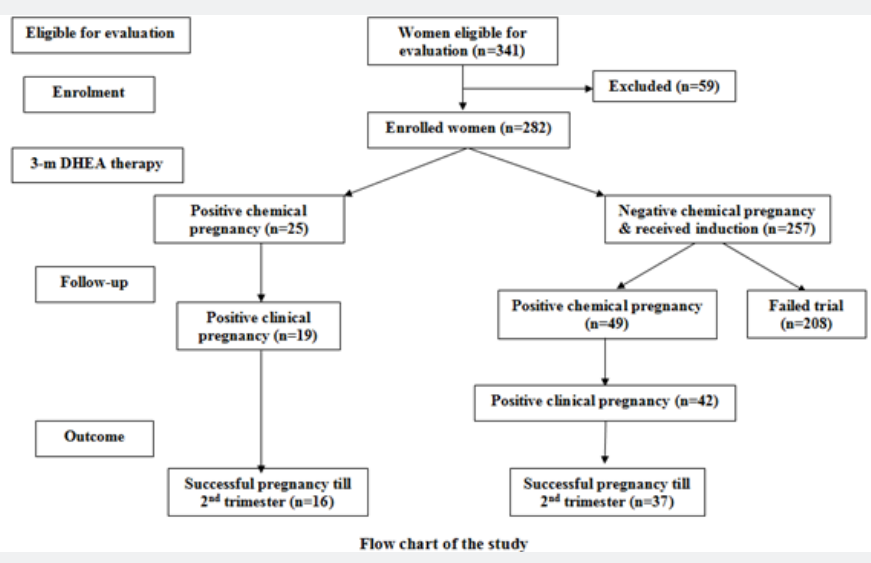

Figure 1: Etiology of Breast cancer.

At the end of phase- 2 of the study, hormonal profile of women included in phase- 2 continued to improve with ovulation induction after 3-m course of DHEA especially serum FSH, LH and E2 that were improved significantly at the end of phase-2 (Figure 2), while serum testosterone and AMH were improved non-significantly compared to levels estimated at the end of phase-1 (Table 3, Figure 3). AFC at the end of phase-2 was significantly

Table 3: Estimated hormonal levels and TUV detected AFC of women included in phase-2 of the study compared to their respective measures at end of phase- 1 of the study..

\begin{tabular}{|c|c|c|c|}
\hline \multirow{2}{*}{ Hormones } & End of phase-1 & End of phase-2 & \multirow{2}{*}{ P value } \\
\cline { 2 - 4 } & (3-m after enrolment) & (6-m after enrolment) & 0.007 \\
\hline FSH $(\mathrm{mIU} / \mathrm{ml})$ & $15.9 \pm 3.3$ & $15 \pm 3.7$ & 0.038 \\
\hline $\mathrm{LH}(\mathrm{mIU} / \mathrm{ml})$ & $6.91 \pm 4.44$ & $6.2 \pm 3.25$ & 0.016 \\
\hline E2 $(\mathrm{pg} / \mathrm{ml})$ & $106.3 \pm 87.6$ & $91.2 \pm 49.3$ & 0.051 \\
\hline Testosterone (ng/ml) & $0.67 \pm 0.39$ & $0.75 \pm 0.48$ & 0.548 \\
\hline AMH (ng/ml) & $1.19 \pm 0.83$ & $1.23 \pm 0.8$ & 0.044 \\
\hline AFC (follicle) & $4.7 \pm 3$ & $5.2 \pm 2.5$ & \\
\hline
\end{tabular}

Data are presented as mean \pm SD; FSH: Follicle stimulating factor; LH: Luteinizing hormone; E2: Estradiol; AMH: anti-Müllerian hormone; AFC: Antral follicle count; $P$ value indicates difference between both groups; $p<0.05$ indicates significant difference 


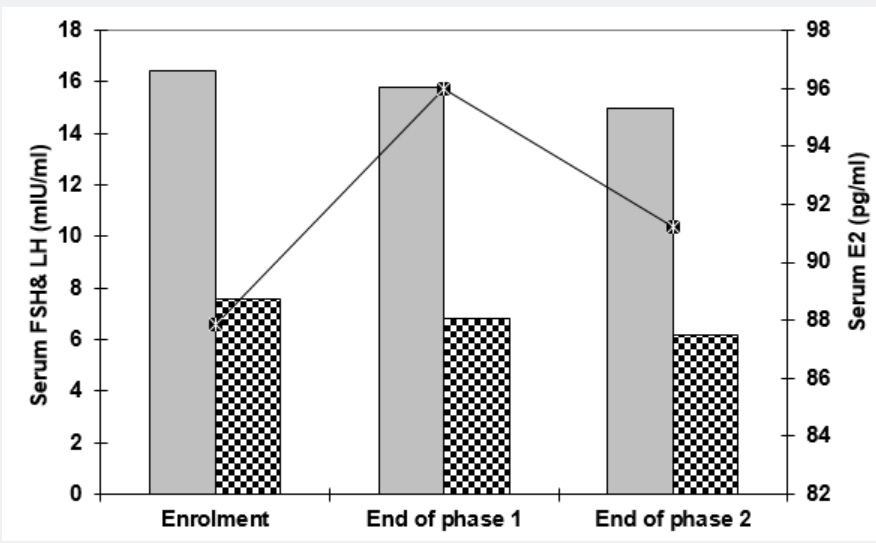

Figure 2: Mean level of FSH, LH and E2 estimated in enrolment and 3-m after DHEA therapy (Phase 1) and 3-m after induction (Phase 2).

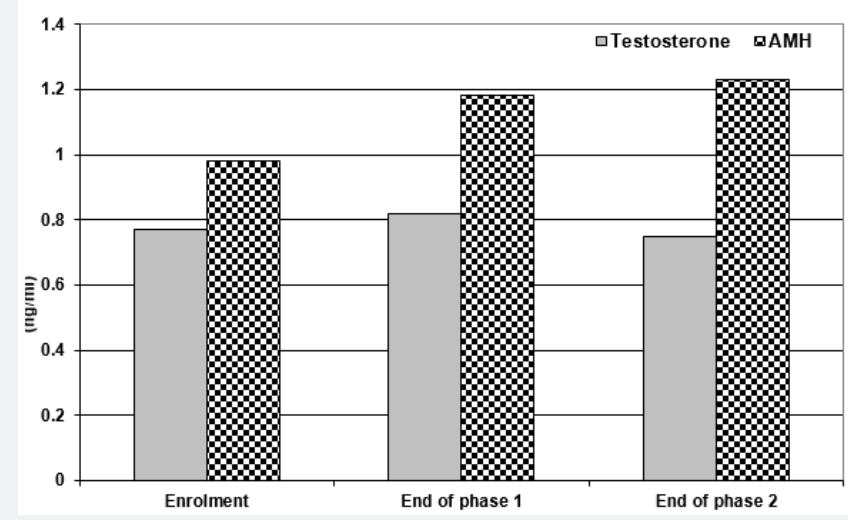

Figure 3: Mean serum level of testosterone \&AMH estimated at enrolment, 3-m after DHEA therapy (Phase 1) and 3-m after induction (Phase 2).

\section{Discussion}

Clinical pregnancy rate, as the study primary outcome, was $21.6 \%$ and success rate for having continued pregnancy till 2nd trimester was $18.8 \%$. Such outcome illustrated the benefit of priming with DHEA with or without induction of ovulation and indicated that the applied protocol could spare the need for assisted reproduction procedures in $21.6 \%$ of infertile women aged $>38$. Moreover, the applied protocol allowed getting pregnancy that was continued till 2nd trimester in $71.6 \%$ of women who had positive chemical pregnancy and $86.9 \%$ of those who had clinical pregnancy. Furthermore, priming by DHEA did favorably with induction of ovulation than without induction as the reported CPR with induction was significantly higher.

Unfortunately, few previous studies tried DHEA for improving the chance of spontaneous conception, but got similar outcome, where Malik et al. [13] reported increased ovulation rate from $48 \%$ to $86.3 \%$ and CPR of $24 \%$ after 3-m DHEA therapy compared to women did not receive DHEA and Agarwal et al. [14] reported significantly higher AFC after DHEA therapy than before treatment and these changes were manifested clinically as improvement in menstrual abnormality and spontaneous conception in $10 \%$ of cases.
The obtained results go in hand with multiple studies tried DHEA for women with POR, prepared for assisted reproduction procedures, where Lin et al. [15] reported greater number of transferred embryos and higher fertilization rate in women aged $>37$ years received DHEA prior to IVF than women who did not receive DHEA. Recently, Chern et al. [16] reported significantly greater number of retrieved, metaphase II and fertilized oocytes with higher CPR in women received DHEA $90 \mathrm{mg} /$ day for 3 months before the IVF cycles than in women had IVF cycles without DHEA. Also, Al-Turki [17] found DHEA supplementation for women with POR undergoing IVF/ICSI cycles significantly improved the endometrial quality, number of retrieved oocytes, quality of embryos, and CPR and live birth rates. Moreover, Schwarze et al. [18] performed meta-analysis for published articles to determine the effect of use of DHEA on the likelihood of success in patients with POR undergoing IVF/ICSI and found DHEA use was associated with significant increase in pregnancy likelihood with significant reduction in the likelihood of abortion, but showed no association with the number of oocytes retrieved.

Secondary outcomes of the current study included changes in AFC and hormonal levels, DHEA therapy induced significant 
reduction of serum FSH, LH with significant increase of testosterone, E2 and AMH levels with subsequently higher AFC compared to pretreatment levels. Similarly, Malik et al. [13] and Agarwal et al. [14] detected significantly improved serum AMH, FSH and E2 levels and AFC. Recently, Al-Turki [17] reported that DHEA supplementation had positive effect on hormonal profiles.

The mechanisms of the beneficial effect of priming of women with POR with DHEA were variable and were extensively studied; experimentally, Chimote \& Chimote [9] documented that DHEA play significantly vital role as intermediate in androgen and estrogen formation and behave as endogenous agonists triggering calcium oscillations required for oocyte activation through regulation of $\mathrm{Ca} 2+$ passage through the calcium channels in oocyte cytoplasm. Chu et al. found supplement DHEA increased the number of primordial and primary follicles in aged mice by inhibiting follicle apoptosis and tended to delay the decrease in levels of cohesion, which may be responsible for age-related aneuploidy in oocytes. Moreover, Eftekhari et al. detected a positive and improvement effect on of DHEA on the meiotic spindle in old mice $[18,19]$.

Clinically, Lin et al. [20], found DHEA treatment resulted in significant reduction of senescence-associated $\beta$-galactosidase, which is was used as marker of senescence in cumulus cells obtained after oocyte retrieval. Hu et al. [19] studied the expression of androgen and FSH receptor in granulosa cells of women received DHEA or not prior to IVF and found DHEA supplementation significantly increased serum levels of testosterone and sulfated DHEA with increased mRNA expression and protein of both receptors in granulosa cells. Lin et al. [20] found DHEA supplementation for women with PORs decreased DNA damage and apoptosis, while enhanced the mitochondrial mass and dehydrogenase activity and transcription factor A expression in cumulus cells.

\section{Conclusion}

Priming of infertile aged women with POR using DHEA with or without ovulation induction improves the chance of spontaneous conception and spares the need for assisted reproduction procedures by $21.6 \%$ of treated women. Ovulation induction after DHEA priming triples the chance for spontaneous conception. However, wider scale multicenter comparative studies are mandatory to establish these outcomes.

\section{References}

1. May Panloup P, Boucret L, Chao de la Barca JM, Desquiret Dumas V, Ferré L Hotellier V, et al. (2016) Ovarian ageing: the role of mitochondria in oocytes and follicles. Hum Reprod Update 22(6): 725-743.

2. Luisi S, Orlandini C, Regini C, Pizzo A, Vellucci F, et al. (2015) Premature ovarian insufficiency: from pathogenesis to clinical management. J Endocrinol Invest 38(6): 597-603.

3. Ben Aharon I, Levi M, Margel D, Yerushalmi R, Rizel S, et al. (2018) Premature ovarian aging in BRCA carriers: a prototype of systemic precocious aging? Oncotarget 9(22): 15931-15941.
4. Huang Y, Hu C, Ye H, Luo R, Fu X, et al. (2019) Inflamm-Aging: A New Mechanism Affecting Premature Ovarian Insufficiency. J Immunol Res 806-9898.

5. Shahrokhi SZ, Kazerouni F, Ghaffari F (2018) Anti-Müllerian Hormone: genetic and environmental effects. Clin Chim Acta 476: 123-129.

6. Dewailly D, Andersen CY, Balen A, Broekmans F, Dilaver N, et al. (2014) The physiology and clinical utility of anti-Mullerian hormone in women. Hum Reprod Update 20(3): 370-385.

7. Nelson SM, Klein BM, Arce JC (2015) Comparison of antimüllerian hormone levels and antral follicle count as predictor of ovarian response to controlled ovarian stimulation in good-prognosis patients at individual fertility clinics in two multicenter trials. Fertil Steril 103(4): 923-930.

8. Dias TR, Alves MG, Almeida SP, Silva J, Barros A, et al. (2015) Dehydroepiandrosterone and 7-oxo-dehydroepiandrosterone in male reproductive health: Implications of differential regulation of human Sertoli cells metabolic profile. J Steroid Bio chem Mol Biol 154: 1-11.

9. Chimote BN, Chimote NM (2018) Dehydroepiandrosterone (DHEA) and Its Sulfate (DHEA-S) in Mammalian Reproduction: Known Roles and Novel Paradigms. Vitam Horm 108: 223-250.

10. Jimenez PT, Frolova AI, Chi MM, Grindler NM, Willcockson AR, et al. (2013) DHEA-mediated inhibition of the pentose phosphate pathway alters oocyte lipid metabolism in mice. Endocrinology 154(12): 48354844 .

11. Triantafyllidou O, Sigalos G, Vlahos N (2017) Dehydroepiandrosterone (DHEA) supplementation and IVF outcome in poor responders. Hum Fertil (Camb) 20(2):80-87.

12. Ferraretti AP, La Marca A, Fauser BC, Tarlatzis B, Nargund G, et al. (2011) ESHRE working group on Poor Ovarian Response Definition. ESHRE consensus on the definition of 'poor response' to ovarian stimulation for in vitro fertilization: The Bologna criteria. Hum Reprod 26: $1616-1624$

13. Malik N, Kriplani A, Agarwal N, Bhatla N, Kachhawa G, et al. (2015) Dehydroepiandrosterone as an adjunct to gonadotropins in infertile Indian women with premature ovarian aging: A pilot study. J Hum Reprod Sci 8(3): 135-141.

14. Agarwal R, Shruthi R, Radhakrishnan G, Singh A (2017) Evaluation of Dehydroepiandrosterone Supplementation on Diminished Ovarian Reserve: A Randomized, Double-Blinded, Placebo-Controlled Study. J Obstet Gynaecol India 67(2): 137-142.

15. Lin LT, Cheng JT, Wang PH, Li CJ, Tsui KH (2017) Dehydroepiandrosterone as a potential agent to slow down ovarian aging. J Obstet Gynaecol Res 43(12): 1855-1862.

16. Chern CU, Tsui KH, Vitale SG, Chen SN, Wang PH, et al. (2018) Dehydroepiandrosterone (DHEA) supplementation improves in vitro fertilization outcomes of poor ovarian responders, especially in women with low serum concentration of DHEA-S: a retrospective cohort study. Reprod Biol Endocrinol 16(1): 90.

17. Al Turki HA (2018) Dehydroepiandrosterone supplementation in women undergoing assisted reproductive technology with poor ovarian response. A prospective case-control study. J Int Med Res 46(1): 143-149.

18. Schwarze JE, Canales J, Crosby J, Ortega Hrepich C, Villa S, et al. (2018) DHEA use to improve likelihood of IVF/ICSI success in patients with diminished ovarian reserve: A systematic review and meta-analysis. JBRA Assist Reprod 22(4): 369-374.

19. Hu Q, Hong L, Nie M, Wang Q Fang Y, et al. (2017) The effect of dehydroepiandrosterone supplementation on ovarian response is associated with androgen receptor in diminished ovarian reserve women. J Ovarian Res 10(1): 32. 
20. Lin LT, Wang PH, Wen ZH, Li CJ, Chen SN, et al. (2017) The Application of Dehydroepiandrosterone on Improving Mitochondrial Function and

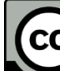

Reducing Apoptosis of Cumulus Cells in Poor Ovarian Responders. Int J Med Sci 14(6): 585-594

\section{Your next submission with Juniper Publishers} will reach you the below assets

- Quality Editorial service

- Swift Peer Review

- Reprints availability

- E-prints Service

- Manuscript Podcast for convenient understanding

- Global attainment for your research

- Manuscript accessibility in different formats ( Pdf, E-pub, Full Tsext, Audio)

- Unceasing customer service

Track the below URL for one-step submission https://juniperpublishers.com/online-submission.php 